\title{
SOME SPLITTING THEOREMS FOR ALGEBRAS OVER COMMUTATIVE RINGS
}

BY

W. C. BROWN

\begin{abstract}
Let $R$ denote a commutative ring with identity and Jacobson radical $p$. Let $\pi_{0}: R \rightarrow R / p$ denote the natural projection of $R$ onto $R / p$ and $j: R / p \rightarrow R$ a ring homomorphism such that $\Pi_{0} j$ is the identity on $R / p$. We say the pair $(R, j)$ has the splitting property if given any $R$-algebra $A$ which is faithful, connected and finitely generated as an $R$-module and has $A / N$ separable over $R$, then there exists an $(R / p)$ algebra homomorphism $j^{\prime}: A / N \rightarrow A$ such that $\Pi_{j^{\prime}}$ is the identity on $A / N$. Here $N$ and $\Pi$ denote the Jacobson radical of $A$ and the natural projection of $A$ onto $A / N$ respectively. The purpose of this paper is to study those pairs $(R, j)$ which have the splitting property. If $R$ is a local ring, then $(R, j)$ has the splitting property if and only if $(R, j)$ is a strong inertial coefficient ring. If $R$ is a Noetherian Hilbert ring with infinitely many maximal ideals such that $R / p$ is an integrally closed domain, then $(R, j)$ has the splitting property. If $R$ is a dedekind domain with infinitely many maximal ideals and $x$ an indeterminate, then the power series ring $R[[x]]$ together with the inclusion map 1 form a pair $(R[[x]], 1)$ with the splitting property. Two examples are given at the end of the paper which show that $R / p$ being integrally closed is necessary but not sufficient to guarantee $(R, j)$ has the splitting property.
\end{abstract}

Introduction. In [3], the notion of a strong inertial coefficient ring $(R, j)$ was first introduced. Let $R$ be a commutative ring with identity. Let $p$ denote the Jacobson radical of $R$ and $\Pi_{0}: R \rightarrow R / p$ the natural projection of $R$ onto $R / p$. We assume there exists a ring homomorphism $j: R / p \rightarrow R$, mapping $R / p$ into $R$, such that $\Pi_{0} j$ is the identity map of $R / p$. Then the pair $(R, j)$ is called a strong inertial coefficient ring if the following property is satisfied: Given any $R$-algebra $A$ which is finitely generated as an $R$-module and has $A / N$ separable over $R$, then there exists an $(R / p)$-algebra homomorphism $j^{\prime}: A / N \rightarrow A$ such that $\Pi j^{\prime}$ is the identity on $A / N$. Here $\Pi$ and $N$ denote the natural projection of $A$ onto $A / N$ and the Jacobson radical of $A$ respectively. In [3], we showed that if $(R, j)$ was a strong inertial coefficient ring, then $R$ itself was an inertial coefficient ring. In [4], the author and E. Ingraham determined the structure of all semilocal inertial coefficient rings. Namely, $R$ is an inertial coefficient ring with finitely many maximal ideals if and only if $R$ is a finite direct sum of Hensel rings. Thus we can characterize semilocal strong inertial coefficient rings as follows: The pair $(R, j)$ is a strong inertial coefficient ring if and only if $R$ is a finite direct sum of split Hensel rings.

Received by the editors June 29, 1970.

AMS 1970 subject classifications. Primary 13B20, 16A16, 16A56; Secondary 13A20, 13F05, 16A21, 16A32.

Key words and phrases. Strong inertial coefficient ring, inertial coefficient ring, splitting property, pair $(R, j)$.

Copyright (C) 1972, American Mathematical Society 
In [3, Proposition 2], we showed that any homomorphic image of a strong inertial coefficient ring was a strong inertial coefficient ring. This property prevents many rings from being strong inertial coefficient rings. For example, the integers $Z$ together with identity map 1 fail to be a strong inertial coefficient ring $(Z, 1)$. This can easily be seen by considering the $Z$-algebra $A=Z / 4 Z$, the integers modulo 4. The radical of $A$ is $2 Z / 4 Z$, and $A / N=Z / 2 Z$ cannot be mapped as $Z$-algebras back into $A$. Thus we are led to the following type of question: If we attempt to modify the definition of a strong inertial coefficient ring in order to avoid examples as the one above, what class of rings will we get? We may avoid examples like $A=Z / 4 Z$ by demanding that $A$ be both faithful and connected over $R$. Thus the purpose of this paper is to study those pairs $(R, j)$ which have the following property: Given any $R$-algebra $A$ which is faithful, connected and finitely generated as an $R$-module, and has $A / N$ separable over $R$, then there exists an $(R / p)$-algebra homomorphism $j^{\prime}: A / N \rightarrow A$ such that $\Pi j^{\prime}$ is the identity on $A / N$. We shall show that in the local case these pairs are exactly strong inertial coefficient rings, but in the nonlocal case we get a much broader class of rings.

Preliminaries. Throughout this paper, all rings will be assumed to be associative and with identities. All ring homomorphisms take the identity to the identity, and all subrings of a given ring contain the identity of that ring. $R$ will always denote a commutative ring with Jacobson radical $p$. By an $R$-algebra $A$, we mean a not necessarily commutative ring $A$ together with a ring homomorphism $\theta$ of $R$ into the center of $A$. If $\theta$ is a monomorphism, we say $A$ is faithful over $R$. If $A$ is faithful over $R$, we shall often just identify $R$ with its image in $A$. We shall say $A$ is finitely generated, projective, etc. over $R$ if $A$ is finitely generated, projective, etc. as an $R$-module. We shall call $A$ connected if the only idempotents of $A$ are 0 and 1 . $N$ will always denote the Jacobson radical of $A$.

Let $\Pi$ and $\Pi_{0}$ denote the projections of $A$ and $R$ onto $A / N$ and $R / p$ respectively. We shall call $R$ split if there exists a ring homomorphism $j: R / p \rightarrow R$ such that $\Pi_{0} j$ is the identity of $R / p$. We shall indicate that $R$ is split by writing the pair $(R, j)$. We shall use the following lemma continually.

Lemma 1. Let $A$ be a finitely generated R-algebra and let $\cap(m A)$ denote the intersection of the $m A$ as $m$ runs over all maximal ideals of $R$. Then

(a) $p A \subseteq N$.

(b) There exists a positive integer $n$ such that $N^{n} \subseteq \bigcap(m A)$.

(c) If $A$ is projective over $R$, then $p A=\bigcap(m A)$.

(d) If $A$ is separable over $R$, then $N=\bigcap(m A)$.

Proof. See [6, Lemma 1.1].

In particular, if $A$ is finitely generated over $R$ and $R$ is split by $j$, then $A / N$ is naturally an $(R / p)$-algebra, and (via $j) A$ is also an $(R / p)$-algebra. We shall say that the pair $(R, j)$ has the splitting property if, given any finitely generated, faithful, 
and connected $R$-algebra $A$ with $A / N$ separable over $R$, there exists an $(R / p)$ algebra homomorphism $j^{\prime}: A / N \rightarrow A$ such that $\Pi j^{\prime}$ is the identity on $A / N$.

Finally, we assume that the reader is familiar with the results of [1] and [6].

1. Semilocal rings with the splitting property. Throughout this section, we shall assume that $R$ is semilocal, i.e. $R$ has only finitely many maximal ideals. If $R$ has more than one maximal ideal, $R / p$ is a finite direct sum of fields. Since $j(R / p) \subset R$, $R$ contains proper idempotents, i.e. idempotents other than 0 or 1 . Hence $(R, j)$ satisfies the splitting property vacuously, for there are no connected, faithful $R$-algebras $A$. Thus any pair $(R, j)$ with $R$ a semilocal ring containing more than one maximal ideal has the splitting property.

Let us now look at the more interesting case when $R$ is local, i.e. $R$ has a unique maximal ideal. In this case, $p$ is just the unique maximal ideal of $R$, and $R / p$ is a field. We make no assumptions concerning chain conditions on $R$.

Suppose $A$ is a finitely generated $R$-algebra via a ring homomorphism $\theta: R \rightarrow A$. If $a_{0}$ is an element of $A$, we denote by $\theta(R)\left[a_{0}\right]$ the subring of $A$ generated by $R$ and $a_{0}$. Thus $\theta(R)\left[a_{0}\right]$ is the subring of $A$ consisting of all polynomials in $a_{0}$ with coefficients in $\theta(R)$.

Lemma 2. Suppose $A$ is a finitely generated algebra over a local ring $R$ (say via $\theta: R \rightarrow A)$. If $a_{0}$ is an element of $A$ such that $\theta(R)\left[a_{0}\right]$ is connected, then $a_{0}$ is a root of a monic polynomial $g(x)$ in $R[x]$ such that $R[x] /(g)$ is connected.

Proof. By $a_{0}$ a root of $g(x)$ in $R[x]$ (x an indeterminate), we of course mean $\theta(g)\left(a_{0}\right)=0$, i.e. if $g(x)=\sum_{i=0}^{n} r_{i} x^{i}, r_{i}$ in $R$, then $\sum_{i=0}^{n} \theta\left(r_{i}\right) a_{0}^{i}=0$ in $A$. Since $A$ is finitely generated over $R$, it follows from [2, Theorem 8] that there exists a monic polynomial $f(x)$ in $R[x]$ such that the ring $R[x] /(f)$ is mapped homomorphically onto $\theta(R)\left[a_{0}\right]$ under the mapping $\zeta: \sum_{i=0}^{m} r_{i} x^{i}+(f(x)) \rightarrow \sum_{i=0}^{m} \theta\left(r_{i}\right) a_{0}^{i}$. Thus if the ring $R[x] /(f)$ is connected we are done. If $R[x] /(f)$ is not connected, then by [2, Lemma 3] there exist monic, relatively prime polynomials $f_{1}(x)$ and $f_{2}(x)$ in $R[x]$ such that $f(x)=f_{1}(x) f_{2}(x)$. Thus

$$
R[x] /(f)=\left(f_{1}\right) /(f) \oplus\left(f_{2}\right) /(f),
$$

i.e. $R[x] /(f)$ decomposes into the direct sum of two mutually orthogonal ideals. Let $\overline{1}$ denote the identity of $R[x] /(f)$. Then from (1), $\overline{1}=e_{1}+e_{2}$, with $e_{i}$ an idempotent in $\left(f_{i}\right) /(f), i=1,2$, and $e_{1} e_{2}=0$. Since $\theta(R)\left[a_{0}\right]$ is connected, $\zeta\left(e_{1}\right)$ is either 0 or 1 . Assume $\zeta\left(e_{1}\right)=1$. Then $\zeta\left(e_{2}\right)=0$ since $\zeta$ is a ring homomorphism. Thus, we have the ring homomorphism

$$
R[x] /\left(f_{2}\right) \stackrel{\aleph}{\longrightarrow}\left\{\frac{R[x]}{(f)}\right\} e_{1} \stackrel{\zeta}{\longrightarrow} \theta(R)\left[a_{0}\right]
$$

with $\aleph$ an isomorphism and $\zeta$ onto. So if $R[x] /(f)$ is not connected, we can find a monic polynomial of smaller degree $f_{2}(x)$ such that $R[x] /\left(f_{2}\right)$ maps onto $\theta(R)\left[a_{0}\right]$ 
under the map sending

$$
\sum_{i=0}^{m} r_{i} x^{i}+\left(f_{2}\right) \rightarrow \sum_{i=0}^{m} \theta\left(r_{i}\right) a_{0}^{i} .
$$

If $R[x] /\left(f_{2}\right)$ is not connected, we continue the above process. After a finite number of steps, we get the desired result.

THEOREM 1. Let $R$ be a local ring split by $j$. Assume the pair $(R, j)$ has the splitting property. If $A$ is any finitely generated, commutative and connected $R$-algebra such that $A / N$ is separable over $R$, then $A$ is a local ring.

Proof. The proof is by contradiction. Since $A$ is finitely generated over $R, A$ is semilocal. Assume $\left\{m_{1}, \ldots, m_{n}\right\}$ are the maximal ideals of $A$ with $n>1$. If we set $F_{i}=A / m_{i}, i=1, \ldots, n, F_{i}$ is a separable field extension of $R / p$. Choose $\alpha$ in $\bigcap_{i=2}^{n} m_{i}$, but not in $m_{1}$. Let $e$ denote the identity of $A$. If $A$ is an $R$-algebra via a ring homomorphism $\theta: R \rightarrow A$, write $r e$ for $\theta(r)$ in $A$. Consider the subring $\operatorname{Re}[\alpha] \subset A, \operatorname{Re}[\alpha]$ the subring of $A$ generated by $\theta(R)$ and $\alpha$. Since $A$ is finitely generated over $R$, $A$ is an integral extension of $\operatorname{Re}$ and thus of $\operatorname{Re}[\alpha]$ also. Therefore, the Jacobson radical of $\operatorname{Re}[\alpha]$ is $N \cap \operatorname{Re}[\alpha]$. Set $\operatorname{rad}(\operatorname{Re}[\alpha])=N \cap \operatorname{Re}[\alpha]$.

We next assert that $\operatorname{Re}[\alpha]$ has exactly two maximal ideals $m_{1} \cap \operatorname{Re}[\alpha]$ and $m_{2} \cap \operatorname{Re}[\alpha]$. Since $A$ is an integral extension of $\operatorname{Re}[\alpha]$, every $m_{i} \cap \operatorname{Re}[\alpha], i=1, \ldots, n$, is maximal in $\operatorname{Re}[\alpha]$. Since $\alpha$ is in $\operatorname{Re}[\alpha], m_{1} \cap \operatorname{Re}[\alpha] \neq m_{2} \cap \operatorname{Re}[\alpha]$. Let $x$ be an element of $m_{2} \cap \operatorname{Re}[\alpha]$. Then $x=r_{0} e+y \alpha$ where $r_{0}$ is in $R$ and $y$ is in $\operatorname{Re}[\alpha]$. Since $\alpha$ and $x$ are in $m_{2}, r_{0} e$ is in $m_{2} \cap R e \subset p e \subset m_{i}, i \geqq 1$. Therefore, $r_{0} e$ and $\alpha$ in $m_{i}$ imply $x$ is in $m_{i} \cap \operatorname{Re}[\alpha], i>1$. Thus $m_{2} \cap \operatorname{Re}[\alpha] \subseteq m_{i} \cap \operatorname{Re}[\alpha], i \geqq 2$. Since $m_{2} \cap \operatorname{Re}[\alpha]$ is maximal in $\operatorname{Re}[\alpha], m_{i} \cap \operatorname{Re}[\alpha]=m_{2} \cap \operatorname{Re}[\alpha], i>1$. Thus $\operatorname{Re}[\alpha]$ has exactly two maximal ideals.

Now $\operatorname{Re}[\alpha] / \operatorname{rad}(\operatorname{Re}[\alpha]) \subset A / N=\bigoplus_{i=1}^{n} F_{i}$. Therefore, $\operatorname{Re}[\alpha] / \operatorname{rad}(\operatorname{Re}[\alpha])$ is the direct sum of two fields which are separable over $R / p$. Thus, $\operatorname{Re}[\alpha] / \operatorname{rad}(\operatorname{Re}[\alpha])$ is separable over $R$. It is easy to see that one of these fields is isomorphic to $R / p$. So we may suppose that

$$
\operatorname{Re}[\alpha] / \operatorname{rad}(\operatorname{Re}[\alpha])=R / p \oplus F_{1} .
$$

Now $\operatorname{Re}[\alpha] \subset A$. Thus $\operatorname{Re}[\alpha]$ is connected. So by Lemma 2, we can find a monic polynomial $f(x)$ in $R[x]$ such that $S=R[x] /(f)$ has the following properties:

1. $S$ is a commutative, finitely generated, free and connected $R$-algebra.

2. $S$ is mapped epimorphically onto $\operatorname{Re}[\alpha]$ under the map sending

$$
\zeta: \sum r_{i} x^{i}+(f) \rightarrow \sum r_{i} e \alpha^{i} .
$$

So we have the following commutative diagram with exact rows:

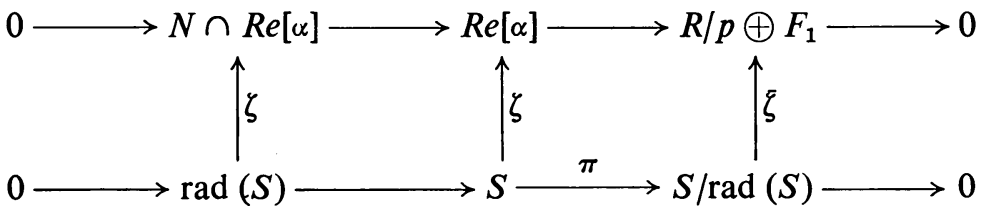


Here $\zeta$ is induced in the natural way from $\zeta$. Since $S$ is finitely generated over $R$, $S / \mathrm{rad}(S)$ is a finite direct sum of fields $L_{1}, \ldots, L_{t}, t \geqq 2$. Since $\zeta$ is onto, one easily checks that one $L_{i}$ is isomorphic to $R / p$ and one $L_{j}, j \neq i$, is isomorphic to $F_{1}$. Let us assume we have labeled things so that $L_{1}=R / p$ and $L_{2}$ is isomorphic to $F_{1}$. Since $F_{1}$ is a finite-dimensional separable field extension of $R / p, F_{1}$ is generated by a primitive element $\bar{a}_{1}$ in $F_{1}$. Choose $\beta$ in $S$ such that $\Pi(\beta)=\bar{a}_{1}$. Then as before, we can easily show that $R[\beta] \subset S$ modulo its radical is isomorphic to $R / p \oplus F_{1}$. Hence $R[\beta]$ is a finitely generated, faithful and connected $R$-algebra ( $S$ is connected) having $R[\beta] / \operatorname{rad}(R[\beta])$ separable over $R$. Since $(R, j)$ has the splitting property, $R[\beta]$ must contain a proper idempotent. This is impossible since $R[\beta]$ is connected. Thus $A$ must be local.

THEOREM 2. Let $(R, j)$ be a pair with $R$ a local ring. Then $(R, j)$ has the splitting property if and only if $R$ is a Hensel ring.

Proof. Suppose $R$ is a Hensel ring. Then by [3, Theorem, 1] $(R, j)$ is a strong inertial coefficient ring. Hence $(R, j)$ clearly has the splitting property.

Suppose $(R, j)$ has the splitting property. By [4, Theorem], in order to show $R$ is Hensel it suffices to show that every finitely generated, commutative and faithful $R$-algebra $S$ with $S / \operatorname{rad}(S)$ separable over $R$ is a finite direct sum of local rings. So let $S$ be such an $R$-algebra. Then we can write $S=S_{1} \oplus \cdots \oplus S_{t}$ as the orthogonal direct sum of algebras $S_{i}$ which satisfy the hypotheses of Theorem 1 . That is, each $S_{i}$ is commutative, connected and finitely generated over $R$ and has $S_{i} / \operatorname{rad}\left(S_{i}\right)$ separable over $R$. Thus the result follows from Theorem 1 .

COROLLARY. If $R$ is a local ring then $(R, j)$ has the splitting property if and only if $(R, j)$ is a strong inertial coefficient ring.

2. Rings with infinitely many maximal ideals and the splitting property. In this section, we assume $R$ has infinitely many maximal ideals and is split by a ring homomorphism $j$. If $R$ is Noetherian, the pair $(R, j)$ cannot be a strong inertial coefficient ring. This follows immediately from the following proposition:

Proposition 1. Suppose the pair $(R, j)$ is a strong inertial coefficient ring. Then if $R$ is Noetherian, $R$ has only finitely many maximal ideals.

Proof. We first assume $p$, the radical of $R$, is 0 . Let $I$ be any ideal of $R$ and consider the algebra $R / I^{2}$ over $R$. Let $\operatorname{rad}\left(R / I^{2}\right)$ denote the Jacobson radical of $R / I^{2}$. Then

$$
0 \longrightarrow \operatorname{rad}\left(R / I^{2}\right) \longrightarrow R / I^{2} \stackrel{\Pi_{0}}{\longrightarrow}\left(R / I^{2}\right) / \operatorname{rad}\left(R / I^{2}\right) \longrightarrow 0
$$

is an exact sequence of $R$-algebras. $R / I^{2}$ is clearly finitely generated over $R$. $\left(R / I^{2}\right) / \mathrm{rad}\left(R / I^{2}\right)$, being a homomorphic image of $R$, is separable over $R$. By hypothesis, $(R, 1)$ is a strong inertial coefficient ring. Hence (1) splits as $R$-algebras, 
i.e. there exists an $R$-algebra homomorphism $j^{\prime}$ of $\left(R / I^{2}\right) / \operatorname{rad}\left(R / I^{2}\right)$ into $R / I^{2}$ such that $\Pi_{0} j^{\prime}=1$. Now $j^{\prime}$ takes the identity to the identity. Therefore

$$
j^{\prime}\left\{\left(R / I^{2}\right) / \operatorname{rad}\left(R / I^{2}\right)\right\}=R / I^{2} .
$$

So $\operatorname{rad}\left(R / I^{2}\right)=0$. Since $I / I^{2} \subset \operatorname{rad}\left(R / I^{2}\right)$, we have $I=I^{2}$. Since $R$ is Noetherian, $I$ is generated by an idempotent [11, Proposition 1.1]. Thus, $I$ is a direct summand of the ring $R$. Since $I$ was arbitrary, this implies that $R$ is a semisimple ring with the descending chain condition. Hence $R$ is a finite direct sum of fields.

Thus if $p=0$ the proposition is proven. In the general case, if $(R, j)$ is a strong inertial coefficient ring, then $(R / p, 1)$ is a strong inertial coefficient ring. Thus $R / p$ is a finite direct sum of fields. This implies $R$ is semilocal.

Thus any Noetherian ring with infinitely many maximal ideals cannot form a strong inertial coefficient ring. However, many of these rings do have the splitting property. We need the following definition:

Definition. We call $R$ a Hilbert ring if every prime ideal of $R$ is the intersection of all maximal ideals containing it.

Clearly any dedekind domain with infinitely many maximal ideals or polynomial rings $k\left[x_{1}, \ldots, x_{n}\right]$ in $n$ indeterminates $x_{i}$ over a field $k$ are examples of Hilbert rings.

Suppose that $R$ is an integral domain which is also a Hilbert ring. Then 0 is a prime ideal and thus the intersection of all the maximal ideals of $R$. Hence $p=0$. The next theorem gives conditions under which $(R, 1)$ has the splitting property.

TheOREM 3. Let $R$ be a Noetherian, integrally closed, Hilbert domain. Then $(R, 1)$ has the splitting property.

Proof. Let $A$ be any finitely generated, faithful, and connected $R$-algebra with $A / N$ separable over $R$. We wish to prove that $0 \rightarrow N \rightarrow A \rightarrow A / N \rightarrow 0$ splits as $R$-algebras. From [7, Corollary 1], $N=L(A)$ the lower radical of $A$. Thus $N$ is the intersection of all prime ideals of $A$. Since $A$ is finitely generated over a Noetherian ring $R, L(A)$ is nilpotent. Hence $N$ is nilpotent.

We next note that $A / N$ is connected. Since $N$ is nilpotent, any idempotent of $A / N$ can be pulled back to an idempotent in $A$. Thus, $A$ connected implies $A / N$ is connected.

Since the radical of $R$ is $0, N \cap R=0$ [2, Corollary, Theorem 9]. Thus $A / N$ is a finitely generated, faithful, connected and separable $R$-algebra. Thus $C(A / N)$, the center of $A / N$, is a finitely generated, faithful, connected, separable and commutative $R$-algebra. Since $R$ is integrally closed, it now follows from [9, Corollary 4.2] that $C(A / N)$ is projective over $R . A / N$ itself is projective over $C(A / N)$. Thus $A / N$ is projective over $R$.

We have now shown that $A$ is a finitely generated $R$-algebra which is a complete Hausdorff space in its $N$-adic topology and has $A / N$ separable and projective over $R$. It follows from [6, Theorem 3.13] that $A$ contains an inertial subalgebra $S$. That 
is, there exists an $R$-separable subalgebra $S$ of $A$ such that $S+N=A$. Now $S$ is finitely generated over $R$ since $R$ is Noetherian. $S$ being separable over $R$ implies $S$ is central separable over $C(S)$, the center of $S$, and $C(S)$ is separable over $R$. Thus $C(S)$ is a commutative, finitely generated, faithful, connected, and separable $R$-algebra. Using [9, Corollary 4.2] again, we get $C(S)$ is projective over $R$. Now by Lemma 1, parts (c) and (d), of this paper, the Jacobson radical, $\operatorname{rad}(C(S))$, of $C(S)$ is generated by the radical of $R$ which is 0 . Therefore $\operatorname{rad}(C(S))=0$. Since $\operatorname{rad}(S) \cap C(S) \subseteq \operatorname{rad}(C(S))$. We get the radical of $S$ when intersected with $C(S)$ is 0 . But $S$ is central separable over $C(S)$. Thus

$$
\operatorname{rad}(S)=[\operatorname{rad}(S) \cap C(S)] S=0 .
$$

So the radical of $S$ is 0 . Since $N \cap S \subseteq \operatorname{rad}(S)$, we have $N \cap S=0$. Thus $A=S \oplus N$ as $R$-algebras. This is equivalent to the existence of an $R$-algebra homomorphism of $A / N$ into $A$ splitting $0 \rightarrow N \rightarrow A \rightarrow A / N \rightarrow 0$.

Thus there are numerous examples of Noetherian domains with infinitely many maximal ideals which can form pairs with the splitting property. For example, $\left(k\left[x_{1}, \ldots, x_{n}\right], 1\right)$ has the splitting property. $(R, 1), R$ a dedekind domain with infinitely many maximal ideals, has the splitting property. In particular, $(Z, 1)$ has the splitting property.

We can also remove the hypothesis that $R$ be a domain.

THEOREM 4. Suppose $R$ is a Noetherian Hilbert ring such that $0 \rightarrow p \rightarrow R \rightarrow R / p$ $\rightarrow 0$ is split by $j$. If $R / p$ is an integrally closed domain, then $(R, j)$ has the splitting property.

Proof. Any homomorphic image of a Hilbert ring is a Hilbert ring. Hence $R / p$ is a Noetherian, integrally closed, Hilbert domain. Let $A$ be any finitely generated, faithful and connected $R$-algebra with $A / N$ separable over $R$. By [7, Corollary 1], both $p$ and $N$ are nilpotent. Since $N \cap R \subset p, A / N$ is faithful over $R / p$. Thus following the procedures of Theorem 3 , we get $A / N$ is projective over $R / p$. So we may view $*: 0 \rightarrow N \rightarrow A \rightarrow A / N \rightarrow 0$ as a short exact sequence of $(R / p)$-algebras with $A / N$ separable, projective and finitely generated over $R / p$. Note $A$ is a complete Hausdorff space in its $N$-adic topology. We may now argue that $*$ splits as $(R / p)$ algebras via induction on the index of nilpotency of $N$.

Let $H_{R / p}^{n}(A / N, N)$ denote the $n$th Hochschild cohomology of $A / N$ coefficients in $N$. Thus, $H_{R / p}^{n}(A / N, N)$ is computed using the bar resolution of $A / N[10$, p. 283]. This makes sense since $A / N$ is projective over $R / p$. Now $A / N$ being separable over $R / p$ implies $H_{R / p}^{1}(A / N, N)=0$, and thus $H_{R / p}^{2}(A / N, N)=0$. Hence if $N$ has index of nilpotency $2, *$ splits as $(R / p)$-algebras via [10, Theorem 3.1, p. 285]. Let us suppose that $*$ splits whenever the index of nilpotency of $N$ is less than $n(n>2)$ and consider the case of $N$ having index $n$. Then $0 \rightarrow N / N^{2} \rightarrow A / N^{2} \rightarrow A / N \rightarrow 0$ is an exact sequence of $(R / p)$-algebras which split by the case $n=2$. Thus there exists an $(R / p)$-subalgebra $T \subset A / N^{2}$ such that $A / N^{2}=N / N^{2} \oplus T$. Hence there exists an 
$(R / p)$-subalgebra $S$ of $A$ such that $S \supset N^{2}$ and $S / N^{2}=T$. Since $T$ is isomorphic to $A / N, T$ is semisimple, i.e. has Jacobson radical 0 . Therefore, the radical of $S$ is $N^{2}$. So $0 \rightarrow N^{2} \rightarrow S \rightarrow A / N \rightarrow 0$ is exact. By our induction hypothesis, this sequence splits as $(R / p)$-algebras. Since $S \subset A$ we get $0 \rightarrow N \rightarrow A \rightarrow A / N \rightarrow 0$ splits as $(R / p)$ algebras.

Thus pairs of the form $\left(k\left[x_{1}, \ldots, x_{n}\right] /\left(x_{i}^{2}\right), 1\right)$ also have the splitting property. One may ask whether $R$ must be a Hilbert ring in order for $(R, j)$ to have the splitting property. The answer to this question is no. To see this, let $R$ be a dedekind domain with infinitely many maximal ideals, e.g. the integers $Z$. Let $x$ be an indeterminate and consider $R[[x]]$-the formal power series ring in $x$, coefficients in $R$. Then $R[[x]]$ is an integrally closed, Noetherian domain with Jacobson radical $(x)$, the ideal generated by $x$. In any Noetherian Hilbert ring, the Jacobson radical is nilpotent. Thus $R[[x]]$ is not a Hilbert ring.

THEOREM 5. $(R[[x]], 1)$ has the splitting property.

Proof. Suppose $A$ is a finitely generated, faithful and connected $R[[x]]$-algebra having $A / N$ separable over $R[[x]]$. Since $A$ is finitely generated over $R[[x]],(x) A \subset N$. Thus, $A / N$ is finitely generated and faithful over $R$.

$A / x A$ is finitely generated over $R$. Thus by [6, Proposition 3.15], the Jacobson radical of $A / x A$, which is $N / A x$, is nilpotent. Hence there exists a positive integer $t$ such that $N^{t} \subset x A$.

Now $R[[x]]$ is a complete Hausdorff space in its $x$-adic topology. Hence $R[[x]]$ is a Zariski ring [12, vol. II, p. 264]. Thus, $\bigcap_{i}(x A)^{i}=0$. So $A$ is a Hausdorff space in its $x A$-adic topology. This implies $A$ is a Hausdorff space in its $N$-adic topology. By [12, Theorem 5, p. 256], $A$ is a complete Hausdorff space in its $x A$-topology and thus in its $N$-adic topology also.

$A$ being a complete Hausdorff space in its $N$-adic topology implies $A$ is suitable for building idempotents. That is, any idempotent of $A / N$ can be lifted to $A$. Thus $A$ connected implies $A / N$ is connected.

Now $A / N$ is a finitely generated, faithful, connected and separable $R$-algebra. Thus using [9, Corollary 4.2] we get the center of $A / N$ projective over $R$. Thus $A / N$ is projective over $R$. Since $A / N$ is projective and separable over $R$, each

$$
0 \rightarrow N / N^{n} \rightarrow A / N^{n} \rightarrow A / N \rightarrow 0 \text { for } n \geqq 2
$$

splits as $R$-algebras. Thus for each $n \geqq 2$ there exists an $R$-separable subalgebra $S_{n}$ of $A / N^{n}$ such that

$$
A / N^{n}=S_{n} \oplus N / N^{n} \text {. }
$$

Following the proof of Theorem 3.16 in [6], we can easily argue that each $S_{n}$ is unique up to an inner automorphism of $A / N^{n}$ generated by an element of $N / N^{n}$. So if $T$ is any other $R$-separable (finitely generated) subalgebra of $A / N^{n}$ such that $T+N / N^{n}=A / N^{n}$, then there exists an element $G$ in $N / N^{n}$ such that

$$
(1-G) T(1-G)^{-1}=S_{n} \text {. }
$$


Let $\Pi_{n+1}: A / N^{n+1} \rightarrow A / N^{n}$ denote the natural projection gotten by factoring out $N^{n} / N^{n+1}$. Then $\Pi_{n+1}\left(S_{n+1}\right)$ and $S_{n}$ are isomorphic via an inner automorphism generated by an element of the form $1-G_{n}, G_{n}$ in $N / N^{n}$. If we lift $G_{n}$ to $G_{n+1}$ in $N / N^{n+1}$, such that $\Pi_{n+1}\left(G_{n+1}\right)=G_{n}$, then $\left(1-G_{n+1}\right) S_{n+1}\left(1-G_{n+1}\right)^{-1}=S_{n+1}^{1}$ has the following properties.

and

$$
S_{n+1}^{1} \oplus N / N^{n+1}=A / N^{n+1}
$$

$$
\Pi_{n+1}\left(S_{n+1}^{1}\right)=\left(1-G_{n}\right) \Pi_{n+1}\left(S_{n+1}\right)\left(1-G_{n}\right)^{-1}=S_{n} .
$$

Hence we may choose the $S_{n}$ at each stage such that $\Pi_{n+1}\left(S_{n+1}\right)=S_{n}$. Then clearly $S=$ proj $\lim S_{n}$, the inverse limit of the $S_{n}$, is an $R$-separable subalgebra of proj $\lim A / N^{n}=A$ such that $S \oplus N=A$. Hence $0 \rightarrow N \rightarrow A \rightarrow A / N \rightarrow 0$ splits as $R$-algebras.

3. Some examples. In this section, we give two examples concerning Theorem 3. One may first ask if the hypothesis that $R$ be integrally closed is necessary. Theorem 3 is false if $R$ is not integrally closed. We see this in the following example which was suggested by $E$. Ingraham.

EXAMPLE. Let $Z$ denote the integers and $S$ the multiplicative set consisting of all powers of a fixed prime $p . S=\left\{p^{i} \mid i=0,1, \ldots\right\}$. We assume $p$ is not equal to 2 or 5 . Let $Z_{S}$ denote the ring $Z$ localized at the multiplicative set $S$. Thus $Z_{S}=\{a|b| a, b$ in $Z$ and $b$ in $S\}$. Let $R=Z_{S}(\sqrt{ } 5)=\left\{x+y \sqrt{ } 5 \mid x, y\right.$ in $\left.Z_{S}\right\}$. Then $R$ is a Noetherian, Hilbert domain with infinitely many maximal ideals. $R$ is not integrally closed. $(1-\sqrt{ } 5) / 2$ is in the quotient field of $R$ and satisfies $x^{2}+x-1$, but $(1-\sqrt{ } 5) / 2$ is not in $R$. We shall show that $(R, 1)$ does not have the splitting property. Let $A=R[x] /\left(x^{2}+x-1\right)$. The discriminant of $x^{2}+x-1$ is 5 . Whence it follows from [9, Theorem 2.2] that $A$ is not a separable $R$-algebra, but $A / m A$ is separable over $R / m$ for all maximal ideals $m$ of $R$ except for that maximal ideal $M$ of $R$ lying over the ideal (5) in $Z_{S}$. Note that $M=\{a+b \sqrt{ } 5 \mid 5$ divides the numerator of $a ; a, b$ in $\left.Z_{S}\right\}$ is the only maximal ideal of $R$ lying over 5 in $Z_{S}$. The radical of $M A$ is

$$
(M A)^{1 / 2}=\left\{a+b x \mid a, b \text { in } M \text { or } a \text { not in } M \text { but } 2 a-b \text { and } a^{2}+b^{2} \text { in } M\right\} .
$$

Denote by $\eta$ the element $(1+\sqrt{ } 5)+2 x$ in $A$. Then $\bar{\eta}=(1-\sqrt{ } 5)+2 x$ and $\eta$ are mutual annihilators. Now $\eta$ is not in $M A$, but $\eta^{2}$ is in $M A$. So $M A \varsubsetneqq M A+A \eta$ $\subseteq(M A)^{1 / 2} \varsubsetneqq A$. By a dimension argument, we see that $M A+A \eta=(M A)^{1 / 2}$. It now follows that the $R$-algebra $A / A \eta$ is $R$-separable, since $(A / A \eta) / M(A / A \eta)$ $=A / M A+A \eta=A /(M A)^{1 / 2}$ is semisimple over the perfect field $R / M$. Separability over the other maximal ideals of $R$ follows from the separability of $A$ over these ideals. Here we are using the well-known result of S. Endo and Y. Watanabe: A finitely generated algebra $A$ over a commutative ring $R$ is separable if and only if $A / m A$ is separable over $R / m$ for all maximal ideals $m$ of $R$.

Thus $A / A \eta$ is separable over $R$. However, $A / A \eta^{2}$ is not $R$-separable, since $\left(A / A \eta^{2}\right) / M\left(A / A \eta^{2}\right)=A / M A$ is not semisimple. 
We note that

and

$$
\begin{aligned}
A \eta= & \{(a+b x)((1+\sqrt{ } 5)+2 x) \mid a, b \text { in } R\} \\
= & \left\{\left(\gamma_{1}+\gamma_{2} \sqrt{ } 5\right)+\left(\gamma_{3}+\gamma_{4} \sqrt{ } 5\right) x \mid-\gamma_{1}+\gamma_{2}+2 \gamma_{4}\right. \\
& \left.=-2 \gamma_{1}+\gamma_{3}+5 \gamma_{4}=0, \gamma_{i} \text { in } Z_{S}\right\}
\end{aligned}
$$

$$
\begin{aligned}
A \bar{\eta}=\left\{\left(\gamma_{1}+\gamma_{2} \sqrt{ } 5\right)\right. & +\left(\gamma_{3}+\gamma_{4} \sqrt{ } 5\right) x \mid \gamma_{1}+\gamma_{2}+2 \gamma_{4} \\
& \left.=-2 \gamma_{1}+\gamma_{3}-5 \gamma_{4}=0, \gamma_{i} \text { in } Z_{S}\right\} .
\end{aligned}
$$

Thus $A \eta \cap A \bar{\eta}=0$ and $A \eta \cap R=0$. So $R \cap A \eta^{2}=0$ and $A / A \eta^{2}$ is a finitely generated, faithful $R$-algebra.

One can show by a straightforward computation that $A \eta$ is a prime ideal of $A$. It is well known that any finitely generated, commutative algebra over a Hilbert ring is a Hilbert ring. Thus $A$ is a Hilbert ring. So $A / A \eta$ is a Hilbert domain and consequently is semisimple. This implies that $A \eta / A \eta^{2}$ is the Jacobson radical of $A / A \eta^{2}$. Thus

$$
*: 0 \rightarrow A \eta / A \eta^{2} \rightarrow A / A \eta^{2} \rightarrow A / A \eta \rightarrow 0
$$

is exact.

We next note that $A / A \eta^{2}$ is connected. For if $e$ is any idempotent in $A / A \eta^{2}$, the image of $e$ in $A / A \eta$ is either 0 or 1 . Thus $e \equiv 0,1 \bmod A \eta / A \eta^{2}$. It now follows from [5, Lemma 1.2] that $e=0$ or 1 . Thus $A / A \eta^{2}$ is connected.

We have now proven that $A / A \eta^{2}$ is a finitely generated, faithful and connected $R$-algebra which is separable modulo its radical $A \eta / A \eta^{2}$. Thus the counterexample will be complete if we show $*$ cannot split as $R$-algebras.

We shall next prove that the $R$-subalgebra $(R+M A) / A \eta^{2}$ is an inertial subalgebra of $A / A \eta^{2}$, i.e. $(R+M A) / A \eta^{2}$ is separable over $R$ and $(R+M A) / A \eta^{2}$ $+A \eta / A \eta^{2}=A / A \eta^{2}$. Recall $M$ lies over 5. Let $m^{\prime}$ be any other maximal ideal of $R$. Then $R+M A+m^{\prime} A=A$ (since $m^{\prime}+M=R$ ), and so $R+M A \rightarrow A \rightarrow A / m^{\prime} A$ is onto. Moreover, $m^{\prime}(R+M A)=\left\{a+b x \mid a\right.$ in $m^{\prime}, b$ in $\left.M m^{\prime}=M \cap m^{\prime}\right\}=(R+M A) \cap m^{\prime} A$, so $(R+M A) / m^{\prime}(R+M A) \cong A / m^{\prime} A$ which is separable over $R / m^{\prime}$. Thus

$$
\left((R+M A) / A \eta^{2}\right) / m^{\prime}\left((R+M A) / A \eta^{2}\right)
$$

is $\left(R / m^{\prime}\right)$-separable for all maximal ideals $m^{\prime} \neq M$ in $R$.

Now. we have

$$
A \eta^{2}=\left\{\left(\gamma_{1}+\gamma_{2} \sqrt{ } 5\right)+\left(\gamma_{3}+\gamma_{4} \sqrt{ } 5\right) x|2| \gamma_{2} 10 \mid \gamma_{3} \text { and } \gamma_{1}, \gamma_{i} \text { in } Z_{S}\right\}
$$

Since $M A$ is generated over $R$ by $5, \sqrt{ } 5,5 x$ and $\sqrt{ } 5 x$, of which the first three are in $M+M^{2} A$, and

$$
\sqrt{ } 5 x=[(-20-8 \sqrt{ } \cdot 5)+(-10-5 \sqrt{ } 5) x]+[(20+8 \sqrt{ } 5)+(10+6 \sqrt{ } 5) x]
$$

is in $M+M^{2} A+A \eta^{2}$, we get $M A \subseteq M+M^{2} A+A \eta^{2}$. Therefore

$$
(R+M A) / A \eta^{2} / M\left((R+M A) / A \eta^{2}\right)=(R+M A) /\left(M+M^{2} A+A \eta^{2}\right)
$$


has dimension one over $R / M$ and thus is separable over $R / M$. We have now shown that $(R+M A) / A \eta^{2}$ is separable over $R$. Also, $(R+M A) / A \eta^{2}+A \eta / A \eta^{2}=A / A \eta^{2}$ since $R+M A+A \eta=R+(M A)^{1 / 2}=A$. So $(R+M A) / A \eta^{2}$ is an inertial subalgebra of $A / A \eta^{2}$.

We next note that $(R+M A) / A \eta^{2}+A \eta / A \eta^{2}$ is not a direct sum, since $(R+M A)$ $\cap A \eta=\{a+b x$ in $A \eta \mid b$ is in $M\} \supsetneq A \eta^{2}$.

Now in a commutative, finitely generated algebra over a Noetherian ring $R$, inertial subalgebras are unique [6, Proposition 2.9]. Thus if $*$ splits as $R$-algebras, $(R+M A) / A \eta^{2}$ would be a direct summand of $A / A \eta^{2}$. This is impossible. Hence $R$ is an example of a Noetherian, Hilbert domain for which $(R, 1)$ does not have the splitting property.

By looking at the theorems in $\$ 2$ of this paper, one could conjecture that any Noetherian, integrally closed domain which splits forms a pair with the splitting property. This is false even in the semisimple case.

EXAMPLE 2. Let $Q$ denote the field of rational numbers, and $x$ and $y$ indeterminates. Let $Q[x]$ denote the polynomial ring of polynomials in $x$, coefficients in $Q$. Let $Q[x]_{(x)}$ denote the ring $Q[x]$ localized at the prime ideal generated by $x$. So

$$
Q[x]_{(x)}=\{g(x) / h(x) \mid g(x), h(x) \text { in } Q[x] \text { and } x \text { does not divide } h(x)\} .
$$

Let $R=\left\{Q[x]_{(x)}\right\}[y]$ the ring of polynomials in $y$ with coefficients in $Q[x]_{(x)}$. Then $R$ is a Noetherian, integrally closed domain. By [8, Theorem 4, p. 12], $R$ is semisimple, i.e. has Jacobson radical $p=0$. We shall show that $(R, 1)$ does not have the splitting property.

Let $A^{1}=R \oplus R /(y) \cong R \oplus Q[x]_{(x)}$. Set $z=(0, x)$ in $A^{1}$. Then $z$ is a quasiregular element of $A^{1}$ which satisfies the equation $z^{2}-x z=0$. Consider the subring $R[z]$ of $A^{1}$. Thus $R[z]$ is the subring of $A^{1}$ consisting of all linear combinations $r_{u}(0, x)^{n}+\cdots+r_{0}$ with $r_{i}$ in $R$. If we let $(z)$ denote the ideal generated by $z$ in $R[z]$, then $(z)$ is the Jacobson radical of $R[z]$. Since $R$ is semisimple, $R[z]=(z) \oplus R$ as $R$-algebras.

We next note that $R[z]$ is connected. For, suppose there exists a $t$ in $R[z]$ such that $t^{2}=t$. Then $t$ can be written uniquely as $g+r$ for $g$ in $(z)$ and $r$ in $R$. Thus

or

$$
g^{2}+2 r g+r^{2}=g+r
$$

$$
g^{2}+2 r g-g=r-r^{2} .
$$

On the left-hand side, we have an element of $(z)$ and on the right an element of $R$. Since $(z) \cap R=0$ we get $r^{2}=r$. Thus $r=0$ or 1 . If $r=0, t=g$ is an element of $(z)$ the radical of $R[z]$. Hence $g$ is both quasiregular and idempotent. Therefore $g=0$. If $r=1, t=g+1$ is a unit in $R[z]$. Hence $t=1$. In either case, we see the only idempotents of $R[z]$ are 0 and 1 . So $R[z]$ is a commutative, finitely generated, faithful and connected $R$-algebra.

Let $X$ be another indeterminate and consider the polynomial $f(X)=X^{2}-(z x-1)$ in $R[z][X]$. Since $z X$ is quasiregular in $R[z], z x-1$ is a unit in $R[z]$. Hence the 
discriminant of $f(X)$ being the unit $4(z x-1)$ in $R[z]$ implies $f(X)$ is a separable polynomial, i.e. $A=R[z][X] /(f(X))$ is a separable $R[z]$-algebra.

We note that $f(X)$ has no roots in $R[z]$. For suppose there existed a $t$ in $R[z]$ such that $t^{2}=z x-1$. If we write $t=g+r$ with $g$ in $(z)$ and $r$ in $R$, we get

$$
g^{2}+2 g r-z x=-r^{2}-1 \text {. }
$$

Thus $r^{2}=-1$ which is impossible. It now follows from [6, Corollary 3.9] that $A$ is connected. So $A=R[z][X] /(f(X))$ is a finitely generated, commutative, connected and faithful $R$-algebra which is both free and separable over $R[z]$.

By Lemma $1, N$ the radical of $A$ is generated by $z$, i.e. $N=z A$. Since $A$ is separable over $R[z], A / N=A / z A$ is separable over $R[z] /(z)=R$. Thus $A$ is a commutative, finitely generated, faithful and connected $R$-algebra such that $A / N$ is separable over $R$. We shall show that $(R, 1)$ does not have the splitting property by showing that $*: 0 \rightarrow N \rightarrow A \rightarrow A / N \rightarrow 0$ does not split as $R$-algebras.

Set $\varepsilon=X+(f(X))$ in $A$. Then $A$ is the free $R[z]$-module on two generators 1 and $\varepsilon$. $\varepsilon$ satisfies the relation $\varepsilon^{2}=z X-1$. Let $\bar{\varepsilon}$ denote the image of $\varepsilon$ in $A / N$. Then $\bar{\varepsilon}^{2}=-1$. Thus if $*$ splits as $R$-algebras, there would exist an element $t$ in $A$ such that $t^{2}=-1$. We shall show that this is impossible. Suppose such a $t$ existed in $A$.

Write $t=l_{1}+l_{2} \varepsilon$ for $l_{1}, l_{2}$ in $R[z]$. Then $t^{2}=-1$ implies

$1^{\circ} .2 l_{1} l_{2}=0$,

$2^{\circ}$. $l_{1}^{2}+l_{2}^{2}(z x-1)=-1$.

Set $l_{1}=g_{1}+r_{1}$ and $l_{2}=g_{2}+r_{2}$ with $g_{i}$ in $(z)$ and $r_{i}$ in $R, i=1,2$. Looking at $1^{\circ}$ we get $2\left(g_{1}+r_{1}\right)\left(g_{2}+r_{2}\right)=0$ or $g_{1} g_{2}+r_{1} g_{2}+r_{2} g_{1}=-r_{1} r_{2}$. Thus

$3^{\circ} . r_{1} r_{2}=0$,

$4^{\circ} . g_{1} g_{2}+r_{1} g_{2}+r_{2} g_{1}=0$.

Since $R$ is a domain, either $r_{1}=0$, or $r_{2}=0$. Now from $2^{\circ}$, we get

$$
\left(g_{1}+r_{1}\right)^{2}+\left(g_{2}+r_{2}\right)^{2}(z x-1)=-1 \text {. }
$$

Separating the part which is in $R$, we get $r_{1}^{2}-r_{2}^{2}=-1$. Thus $r_{2} \neq 0$. Therefore $r_{1}=0$ and $r_{2}= \pm 1$.

In either case, $l_{2}=g_{2} \pm 1$ is a unit in $R[z]$. Hence $1^{\circ}$ implies $l_{1}=0$. Thus we are down to an equation of the form $(g \pm 1)^{2} \varepsilon^{2}=-1$ with $g$ an element of $(z)$ in $R$.

Suppose $r_{2}=1$. Then we have $(g+1)^{2}(z x-1)=-1$. Since $z^{2}=z x$ we get $(g+1)^{2}\left(z^{2}-1\right)=-1$ or

$5^{\circ}$. $\left(g^{2}+2 g+1\right) z^{2}=g^{2}+2 g$ in $R[z]$.

Now $g$ in $(z)$ has the form $a_{n} z^{n}+\cdots+a_{1} z$ where the $a_{i}$ are polynomials in $y$ with coefficients in $Q[x]_{(x)}$. Thus $5^{\circ}$ becomes

$$
\begin{aligned}
\left\{\left[a_{n}(0, x)^{n}+\cdots+a_{1}(0, x)\right]^{2}+2\left[a_{n}(0, x)^{n}+\cdots+a_{1}(0, x)\right]+1\right\}\left(0, x^{2}\right) \\
=\left[a_{n}(0, x)^{n}+\cdots+a_{1}(0, x)\right]^{2}+2\left[a_{n}(0, x)^{n}+\cdots+a_{1}(0, x)\right] .
\end{aligned}
$$

Counting degrees in $x$, we see that $y$ must divide $a_{n}$, i.e. $a_{n}$ has no constant term in $Q[x]_{(x)}$. Thus $a_{n}(0, x)^{n}=0$. Continuing in this way, we get $a_{i}(0, x)^{i}=0, i=1, \ldots, n$. Therefore $g=0$ which is impossible. The case $r=-1$ is similar. 
Thus the ring $R=Q[x]_{(x)}[Y]$ is an example of a Noetherian, integrally closed domain with radical 0 for which the pair $(R, 1)$ does not have the splitting property.

\section{REFERENCES}

1. M. Auslander and $\mathrm{O}$. Goldman, The Brauer group of a commutative ring, Trans. Amer. Math. Soc. 97 (1960), 367-409. MR 22 \#12130.

2. G. Azumaya, On maximally central algebras, Nagoya Math. J. 2 (1951), 119-150. MR 12, 669.

3. W. C. Brown, Strong inertial coefficient rings, Michigan Math. J. 17 (1970), 73-84.

4. W. C. Brown and E. C. Ingraham, A characterization of semilocal inertial coefficient rings, Proc. Amer. Math. Soc. 26 (1970), 10-14. MR 41 \#5354.

5. S. Greco, Algebras over nonlocal Hensel rings, J. Algebra 8 (1968), 45-59. MR 36 \#1435.

6. E. C. Ingraham, Inertial subalgebras of algebras over commutative rings, Trans. Amer. Math. Soc. 124 (1966), 77-93. MR 34 \#209.

7. - On the occasional equality of the lower and Jacobson radicals in noetherian rings, Arch. Math. (Basel) 20 (1969), 267-269. MR 39 \#5616.

8. N. Jacobson, Structure of rings, Amer. Math. Soc. Colloq. Publ., vol. 37, Amer. Math. Soc., Providence, R. I., 1964. MR 36 \#5158.

9. G. J. Janusz, Separable algebras over commutative rings, Trans. Amer. Math. Soc. 122 (1966), 461-479. MR 35 \#1585.

10. S. Mac Lane, Homology, Die Grundlehren der math. Wissenschaften, Band 114, Academic Press, New York; Springer-Verlag, Berlin, 1963. MR 28 \#122.

11. W. Vasconcelos, On finitely generated flat modules, Trans. Amer. Math. Soc. 138 (1969), 505-512. MR 39 \#199.

12. O. Zariski and P. Samuel, Commutative algebra. Vol. II, University Series in Higher Math., Van Nostrand, Princeton, N. J., 1960. MR 22 \#11006.

Michigan State University, East Lansing, Michigan 48823 